\title{
APLICAÇÃO DA TÉCNICA DE ELETROCOAGULAÇÃO PARA A REMOÇÃO DA CORANTE TÊXTIL
}

\author{
Alessandra Algeri ${ }^{1}$, Augusto Vaghetti Luchese ${ }^{2}$, Pamella Mendes Morini ${ }^{3}$ e \\ Elizandra Sehn ${ }^{4}$
}

\begin{abstract}
Resumo: O objetivo deste trabalho consistiu em determinar o tempo operacional e a densidade de corrente elétrica que otimizassem a remoção da cor de um efluente sintético à base de corante vermelho reativo, por Delineamento Composto Central Rotacional (DCCR). A partir das condições otimizadas foi realizada uma análise de custos operacionais em função da energia gasta e também do consumo de eletrodo. As condições ótimas e os custos operacionais foram determinados para os comprimentos de onda de 515 e $543 \mathrm{~nm}$. A região ótima de remoção, para os dois comprimentos de onda, ficou entre 47 e 51,1 minutos e 360-440 A m${ }^{-2}$, com valores teóricos de remoção estimados entre 70 e $80 \%$. O consumo de eletrodos e de energia nos tempos e densidade de correntes otimizados encontrados ficou em $1,71 \mathrm{~g} \mathrm{~L}^{-1}$ e $0,0077 \mathrm{Wh} \mathrm{m} \mathrm{m}^{-3}$ para o comprimento de onda de 515 $\mathrm{nm}$ e 1,73 $\mathrm{g} \mathrm{L}^{-1}$ e 0,0073 $\mathrm{Wh} \mathrm{m}^{-3}$ para o de $543 \mathrm{~nm}$, resultando nas equações: $C=0,0077 \mathrm{a}+1,71 \mathrm{~b}$, para o comprimento de $515 \mathrm{~nm}$ e $\mathrm{C}=0,0073 \mathrm{a}+1,73 \mathrm{~b}$ para o $543 \mathrm{~nm}$, nos quais a corresponde ao custo de energia (em $R \$ . \mathrm{kWh}^{-1}$ ) e b ao custo mássico da placa (em $R \$ . \mathrm{kg}_{\text {eletrodo-1). }}{ }^{-1}$.
\end{abstract}

Palavras-chave: Densidade de corrente. Corante têxtil. Otimização. Tempo operacional.

\section{Introdução}

Dentro da grande gama de resíduos gerados nas mais diversas indústrias, os efluentes líquidos merecem atenção especial devido à fácil difusão dos poluentes e contaminantes, além dos danos causados à saúde, aliados à difícil manutenção da qualidade ambiental. Neste âmbito, as pesquisas sobre 0 tratamento de efluentes têm avançado bastante na tentativa de se obter uma técnica que seja eficiente tanto energética, econômica como ambientalmente (GOBBI, 2013).

As indústrias têxteis geram grande quantidade de efluentes industriais, já que o volume de água consumido no processo de tingimento é muito grande (NASCIMENTO et al., 2014; KELM et al., 2014). Exemplos de características marcantes dos efluentes têxteis são a coloração acentuada e as elevadas cargas orgânica e inorgânica, causadas pelo processo de acabamento e tingimento (AQUINO NETO et al., 2011).

A grande maioria das indústrias consegue realizar 0 tratamento de seus

sandra.algeri.utfpr@gmail.com

UFPR - R. Pioneiro, 2153 - Dallas, Palotina - PR, 85950-000

\footnotetext{
2 aluchese@gmail.com

3 pamellamorini@hotmail.com

4 elizandrasehn@hotmail.com
}

efluentes líquidos através de tratamento biológico. Entretanto, algumas indústrias, como as têxteis, não podem utilizar este tipo de tratamento, porque seus efluentes possuem elevada recalcitrância, devido à presença de moléculas de corantes (PARENTE et al., 2015; FLECK; TAVARES; EYNG, 2013; VASQUES et al., 2011).

Os corantes reativos são altamente recalcitrantes, colorantes e com potencial cancerígeno e tóxico. Desta maneira, devem ser removidos dos efluentes de indústrias têxteis para que causem o menor impacto sobre o meio ambiente (SENTHILKUMAAR et al., 2006). A remoção de corante vermelho reativo foi estudada por Elkady, Ibrahim, e ElLatif (2011), a partir da adsorção. Entretanto, o equilíbrio da solução foi alcançado somente após três horas de reação e com pH muito baixo. A degradação de corante por processos oxidativos avançados (POAs), como o ozônio, não foi completamente eficiente para remoção do corante vermelho reativo. Logo, foi necessário também o ajuste do pH (SANTOS et al., 2011) que, juntamente 


\section{REA - Revista de estudos ambientais (Online)}

v.19, n. 2, p.31-39, jul./dez. 2017

com a utilização do ozônio, eleva muito o custo deste tipo de tratamento.

Uma alternativa interessante para o tratamento de efluentes têxteis é a técnica da eletrocoagulação, que consiste na passagem de corrente elétrica por um ânodo de sacrifício (ferro ou alumínio), posicionado no tanque de processo (CERQUEIRA; MARQUES, 2011). A passagem da corrente faz com que sejam liberados íons metálicos que passam a atuar como coagulantes da matéria orgânica. Desde processo também resultam a formação de gases que irão promover a flotação das partículas aderidas nos coágulos (VALERO et al., 2007). A eletrocoagulação vem sendo estudada para o tratamento de efluente têxtil (PASCHOAL; TREMILIOSI-FILHO, 2005; VALERO et al., 2007; PALÁCIO et al., 2015; ALEXANDRE et al., 2014). Estes estudos revelaram que essa técnica é eficiente e podem ser observados valores de remoção superiores a $90 \%$ do corante em efluentes sintéticos (VALERO et al., 2008).

O processo de eletrocoagulação ocorre em três fases. A primeira trata-se da formação do agente coagulante, por oxidação eletrolítica do eletrodo de sacrifício (Fe/Al) que promove a desestabilização dos coloides. $\mathrm{Na}$ sequência ocorre a aglutinação das partículas coloidais, para a formação de flocos, promovida pelos hidróxidos de ferro e alumínio que são bons coagulantes naturais e, por fim, a formação dos gases $\mathrm{O}_{2}$ e $\mathrm{H}_{2}$, no ânodo e no cátodo respectivamente. Esses fazem com que os flocos que se formaram sejam arrastados até a superfície do reator, resultando assim no efluente clarificado (CERQUEIRA, 2006).

A técnica de eletrocoagulação possui algumas vantagens se comparada com outras técnicas de tratamento de efluentes tais como a simplicidade de equipamentos, a fácil remoção dos flocos, a aglutinação de coágulos menores e a remoção de compostos recalcitrantes (MOLLAH et al., 2001).

Em qualquer tratamento de efluentes, um fator extremamente importante é o custo associado ao método. A eletrocoagulação tem custo um tanto elevado. E, cerca de $80 \%$ do custo total do tratamento são associados ao consumo de material. Neste caso, os eletrodos e, principalmente, o custo com energia elétrica (GOBBI, 2013).

Assim, o presente estudo teve como objetivo encontrar a melhor condição de remoção do corante vermelho reativo $4 \mathrm{~B}$ pelo processo de eletrocoagulação, utilizando eletrodos de ferro. Para tanto, o método de Delineamento Composto Central Rotacional (DCCR) foi utilizado para determinar uma região ótima de trabalho para as variáveis: tempo de tratamento e a densidade de corrente elétrica. Também foi analisado o custo de tratamento para a condição ótima encontrada.

\section{Metodologia}

\subsection{Solução sintética de corante vermelho reativo}

A técnica de eletrocoagulação foi utilizada para avaliar a remoção da cor de uma solução sintética preparada com 0 corante vermelho reativo $4 \mathrm{~B}$. Para tanto foram adicionados $50 \mathrm{mg}$ do corante diluídos em $1 \mathrm{~L}$ de água destilada. A fim de aumentar a condutividade, também foram adicionados $4 \mathrm{~g}$ de $\mathrm{NaCl}$ à solução.

\subsection{Planejamento experimental}

Um delineamento Composto Central Rotacional (DCCR) foi selecionado para avaliar as variáveis independentes: tempo operacional e densidade de corrente. O parâmetro utilizado para a observação da eficiência do tratamento foi a remoção da cor. Foram realizados 4 ensaios fatoriais, 4 repetições centrais e mais 4 ensaios axiais, totalizando 12 ensaios, todos realizados em duplicata.

Determinar uma condição ótima de tratamento é extremamente importante, devido ao fator econômico associado à técnica de eletrocoagulação. Como se utiliza energia elétrica é fundamental determinar o tempo e a corrente que viabilizem a utilização desta técnica.

Os valores reais de tempo e de corrente, utilizados na matriz DCCR correspondentes aos valores codificados, estão apresentados na Tabela 1. Para chegar aos valores de 15 e 45 minutos e nas densidades de corrente de 200 e $400 \mathrm{~A} \mathrm{~m}^{-2}$ foram realizados alguns testes preliminares, a fim de verificar quais eram as faixas de estudo de tempo operacional e densidade de corrente adequadas para a realização do experimento. 


\section{REA - Revista de estudos ambientais (Online) v.19, n. 2, p.31-39, jul./dez. 2017}

Tabela 1 - Valores reais de tempo e de corrente utilizados na matriz de delineamento

\begin{tabular}{cccccc}
\hline Parâmetros & $\mathbf{- 1 , 4 1}$ & $\mathbf{- 1}$ & $\mathbf{0}$ & $\mathbf{1}$ & $\mathbf{1 , 4 1}$ \\
\hline Densidade de corrente $\left(\mathrm{A} \mathrm{m}^{-2}\right)$ & 160 & 200 & 300 & 400 & 440 \\
Tempo operacional $(\mathrm{min})$ & 8,8 & 15 & 30 & 45 & 51,1 \\
\hline
\end{tabular}

Fonte: Autores (2017)

Ao realizar um DCCR obtém-se uma equação de segundo ou de primeiro grau que gera uma superfície de resposta da variável dependente, neste caso a remoção da cor, em função das variáveis independentes (tempo operacional e densidade de corrente). Com base nesta equação é possível determinar a região ótima de operação para as variáveis analisadas. A equação 1 discrimina o modelo geral para a remoção da cor.

$$
y=\bar{y}+a_{1} i+a_{2} i^{2}+a_{3} t+a_{4} t^{2}+a_{5} i t
$$

Em que:

y = representa a variável resposta, no caso, a percentagem de remoção da cor;

$\bar{y}=$ constante de interceptação;

$a_{1, . ., 5}=$ coeficientes de regressão lineares e quadráticos;

$\mathrm{i}, \mathrm{t}=$ variáveis independentes, tempo operacional e densidade de corrente.

\subsection{Eletrocoagulação e parâmetros físico- químicos}

O procedimento aconteceu em reatores de $1000 \mathrm{ml}$, e cada um dos reatores contou com $500 \mathrm{ml}$ da solução a ser tratada. Os eletrodos utilizados foram de ferro com 0,6 $\mathrm{mm}$ de espessura e área ativa (submersa) de $2500 \mathrm{~mm}^{2}$. Os eletrodos foram separados com material isolante de forma que a distância entre eles fosse de $1 \mathrm{~cm}$. Os ensaios de eletrocoagulação ocorreram em batelada e os eletrodos foram conectados a uma fonte de alimentação modelo FA-3050 e marca Instrutherm, com cada eletrodo conectado a um polo diferente (positivo e negativo). Os ensaios foram mantidos sob agitação constante por agitadores magnéticos.

A medida de cor foi feita por um espectrofotômetro da marca Hach - Modelo DR 2800, antes e depois do tratamento com eletrocoagulação, a fim de identificar a eficiência do processo, bem como o comportamento da solução tratada e a porcentagem de remoção foi calculada de acordo com a equação 2. Antes das medições de cor o espectrofotômetro foi calibrado para a faixa de leitura (0-50 $\mathrm{mg} \mathrm{L}^{-1}$ do corante vermelho reativo). Após o tratamento, as amostras foram retiradas depois de uma hora de decantação com o auxílio de uma pipeta. A determinação da medida da cor foi feita em dois comprimentos de onda (515 e $545 \mathrm{~nm}$ ), característicos do corante vermelho reativo, identificados pela leitura da solução de corante bruta no espectro de varredura da marca Perkin Elmer.

$$
\% \operatorname{Rem}=\frac{(\mathrm{Lb}-\mathrm{La})}{\mathrm{Lb}} \cdot 100
$$

Em que, Lb é a leitura do efluente sintético bruto, sem tratamento, La é a leitura da amostra tradada de acordo com a matriz de ensaio (Tabela 2) e \% Rem é o percentual de remoção da cor

\subsection{Consumo de eletrodos e energia}

Após a determinação da região ótima de tratamento, foram realizados ensaios com o tempo operacional e a densidade de corrente ótimos para validar os resultados teoricamente obtidos pelo DCCR. Durante esses testes, foi efetuada a medida da massa do eletrodo antes e após o tratamento da eletrocoagulação, com o intuito de obter um valor da massa de eletrodo consumida durante a eletrólise. Cabe ressaltar que a limpeza dos eletrodos após o tratamento foi feita somente com água destilada e estes foram secos em temperatura ambiente e posteriormente sua massa foi mensurada em uma balança analítica. Os valores da diferença de potencial (V) utilizados no processo de tratamento também foram coletados. A massa do eletrodo consumida $\left(\mathrm{C}_{\mathrm{el}}\right)$ teoricamente durante a eletrólise foi quantificada de acordo com a equação 3.

$$
\mathrm{C}_{\mathrm{el}}=\frac{\mathrm{i} \times \mathrm{x} \times \mathrm{M}}{\mathrm{F} \times \mathrm{n}}
$$

Em que i é a densidade de corrente (A); t, o tempo operacional (s); M, a massa molar do elemento predominante do eletrodo ( $\mathrm{g} \mathrm{mol}^{-1}$ ); $\mathrm{n}$, o número de elétrons envolvidos na reação de oxidação do elemento do ânodo; e F, a constante de Faraday $\left(9,65 \times 10^{4} \mathrm{C} \mathrm{mol}^{-}\right.$ 1) (CERQUEIRA, 2006). Já o consumo de energia foi calculado de acordo com a Equação 4 (KOBYA et al., 2006; GOBBI, 2013). 
REA - Revista de estudos ambientais (Online)

v.19, n. 2, p.31-39, jul./dez. 2017

$$
C=\frac{U \times i \times t^{\prime}}{V}
$$

Em que $\mathrm{C}$ energia é o consumo de energia $\left(\mathrm{kWh} \mathrm{m}^{-3}\right)$; U é a tensão elétrica aplicada no sistema (V); i é a corrente elétrica aplicada (A); t, o tempo de aplicação da corrente (h); e V, o volume de efluente tratado $\left(\mathrm{m}^{3}\right)$. O correto para contabilizar os custos totais é levar em consideração o consumo dos eletrodos e de energia. Assim, a equação 5 estima adequadamente o custo operacional (GOBBI, 2013).

$$
\mathrm{C}_{\mathrm{O}}=\mathrm{aC}+\mathrm{bC} \mathrm{El}_{\mathrm{E}}
$$

Onde: $C_{O}$ é o custo de operação $(R \$$ $\mathrm{m}^{-3}$ efluente); a, o custo de energia ( $\mathrm{R} \$ \mathrm{kWh}^{-}$ $\left.{ }^{1}\right)$; C, o consumo de energia ( $\mathrm{kWh} \mathrm{m}^{-3}$ efluente); b, o custo mássico da placa $(R \$ \mathrm{~kg}$ $\left.{ }^{1}\right)$; e $C_{E l}$, o consumo do eletrodo $\left(\mathrm{kg} \mathrm{m}^{-3}\right.$ efluente).

\subsection{Análises estatísticas}

Foi utilizado o programa Statística versão 7 para a análise estatística dos dados e obtenção dos parâmetros significativos nas análises desenvolvidas. É importante ressaltar que todos os cálculos estatísticos, obtenção dos coeficientes e do consequente ótimo percentual de remoção corante, foram realizados para os dois comprimentos de onda, característicos do corante vermelho reativo, os quais são 515 e 543 nm.

\section{Resultados e discussão}

Os valores de percentual de remoção do corante vermelho reativo para os ensaios da matriz do DCCR estão apresentados na Tabela 2. É importante salientar que os valores de remoção são médios, em virtude da duplicidade de cada teste realizado.

Tabela 2 - Remoção de cor para cada ensaio do DCCR com os respectivos valores codificados utilizados em cada ensaio

\begin{tabular}{ccccc}
\hline Matriz & $\begin{array}{c}\text { Densidade } \\
\text { de corrente }\end{array}$ & $\begin{array}{c}\text { Tempo } \\
\text { operacional }\end{array}$ & $\begin{array}{c}\text { Remoção (\%) } \\
\text { Rm }\end{array}$ & $543 \mathrm{~nm}$ \\
\hline 1 & 1 & 1 & 69 & 76 \\
2 & 1 & -1 & 46 & 54 \\
3 & -1 & 1 & 47 & 55 \\
4 & -1 & -1 & 41 & 46 \\
5 & 0 & 0 & 59 & 66 \\
6 & 0 & 0 & 55 & 63 \\
7 & 0 & 0 & 49 & 55 \\
8 & 0 & 0 & 59 & 64 \\
9 & 1,41 & 0 & 56 & 62 \\
10 & $-1,41$ & 0 & 38 & 44 \\
11 & 0 & 1,41 & 64 & 70 \\
12 & 0 & $-1,41$ & 43 & 47 \\
\hline
\end{tabular}

Fonte: Autores (2017)

As remoções da cor para ambos os comprimentos de onda (Tabela 2) foram semelhantes e foram numericamente maiores no primeiro ensaio, com tempo operacional de 45 minutos e densidade de corrente de $400 \mathrm{~A}$ $\mathrm{m}^{-2}$. Os valores de percentual de remoção encontrados neste estudo estão abaixo dos obtidos por Valero et al. (2008) que trabalharam com eletrocoagulação na remoção do corante vermelho remazol e encontraram até $99 \%$ de remoção da cor. Mas vale ressaltar que esses autores empregaram uma intensidade de corrente de
2,5 A, acima da intensidade de corrente de 1,1 A utilizada neste estudo, e eletrodos de alumínio e não de ferro.

Na Tabela 3, estão apresentados os valores referentes ao tratamento estatístico dos dados do comprimento de $515 \mathrm{~nm}$. Podese observar que os efeitos de todos os fatores foram considerados significativos, pois apresentaram o valor de $p$ menor do que 0,05. O termo linear da corrente, o termo linear do tempo e o termo de interação entre corrente e tempo apresentaram valores positivos. Tais dados indicam que, ao passar do nível inferior 


\section{REA - Revista de estudos ambientais (Online) v.19, n. 2, p.31-39, jul./dez. 2017}

para o nível superior, a remoção de cor é mais elevada. Já os outros termos apresentaram efeito negativo, ou seja, a passagem do nível inferior para o nível superior dos ensaios resultou em decréscimo da remoção de cor.

Tabela 3 - Efeitos dos termos referentes ao modelo de remoção de cor para comprimento de

\begin{tabular}{ccccc}
\multicolumn{5}{c}{$\mathbf{5 1 5} \mathbf{~ n m}$} \\
\hline Fatores & Efeito & $\begin{array}{c}\text { Erro } \\
\text { padrão }\end{array}$ & $\mathbf{t}(\mathbf{5})$ & p-valor \\
\hline Média & 57,6645 & 0,8794 & 65,5690 & $0,0000^{*}$ \\
(1)Corrente (L) & 13,1341 & 1,0787 & 12,1756 & $0,0001^{*}$ \\
Corrente (Q) & $-10,4545$ & 1,2872 & $-8,1220$ & $0,0005^{*}$ \\
(2) Tempo (L) & 14,6962 & 1,0787 & 13,6238 & $0,0000^{*}$ \\
Tempo (Q) & $-3,9156$ & 1,2872 & $-3,0420$ & $0,0287^{*}$ \\
1L versus 2L & 8,5000 & 1,5233 & 5,5801 & $0,0025^{*}$
\end{tabular}

* Termos significativos a um nível de significância de 0,05

Fonte: Autores (2017)

$\mathrm{Na}$ Tabela 4 estão os valores estatísticos referentes aos fatores do comprimento de $543 \mathrm{~nm}$. Assim como para o comprimento de $515 \mathrm{~nm}$, todos os termos do modelo apresentaram efeitos significativos, pois o p-valor apresentado foi menor que 0,05 . Os termos lineares da corrente e do tempo apresentaram efeitos positivos bem como o termo de interação entre a corrente e o tempo. Isso indica que a passagem do nível inferior para o nível superior dessas variáveis promove o aumento da remoção de cor na solução com o corante vermelho.

Tabela 4 - Dados estatísticos referentes ao comprimento de $543 \mathrm{~nm}$

\begin{tabular}{ccccc}
\hline Fatores & Efeito & $\begin{array}{c}\text { Erro } \\
\text { padrão }\end{array}$ & $\mathrm{t}(5)$ & $\mathrm{p}$-valor \\
\hline Média & 64,3252 & 0,9953 & 64,6278 & $0,0000^{*}$ \\
(1) Corrente (L) & 13,6356 & 1,2208 & 11,1690 & $0,0001^{*}$ \\
Corrente (Q) & $-10,3612$ & 1,4568 & $-7,1124$ & $0,0009^{*}$ \\
(2) Tempo (L) & 15,9048 & 1,2208 & 13,0277 & $0,0000^{*}$ \\
Tempo (Q) & $-4,8283$ & 1,4568 & $-3,3143$ & $0,0211^{*}$ \\
1L versus 2L & 6,5000 & 1,7240 & 3,7704 & $0,0130^{*}$ \\
\hline
\end{tabular}

* Termos significativos a um nível de significância de 0,05

Fonte: Autores (2017)

$\mathrm{Na}$ Tabela 5 está apresentada a análise de variância realizada para validação dos modelos de remoção de cor, em que foram utilizados todos os termos significativos, e o limite de confiança adotado foi de 0,95 para os comprimentos de onde de 515 e $543 \mathrm{~nm}$.

Tabela 5 - Anova para validação dos modelos matemáticos para os comprimentos de 515 e $543 \mathrm{~nm}$

\begin{tabular}{|c|c|c|c|c|c|c|c|c|}
\hline $\begin{array}{l}\text { Comprimento } \\
\text { de onda }(\mathrm{nm})\end{array}$ & $\begin{array}{c}\text { Fonte de } \\
\text { Var. }\end{array}$ & $\mathrm{SQ}^{1}$ & $\mathrm{GL}^{2}$ & $M Q^{3}$ & $\begin{array}{c}\mathbf{F} \\
\text { calculado }\end{array}$ & F tabelado & p-valor & $\mathbf{R}^{2}$ \\
\hline & Regressão & 1001,13 & 5 & 200,23 & 86,3 & 5,05 & 0,0026 & $98,8 \%$ \\
\hline \multirow[t]{3}{*}{515} & Resíduos & 11,60 & 5 & 2,32 & & & & \\
\hline & Total & 1012,727 & 10 & & & & & \\
\hline & Regressão & 1072,78 & 5 & 214,56 & 72,2 & 5,05 & 0,0026 & $98,6 \%$ \\
\hline \multirow[t]{2}{*}{543} & Resíduos & 14,86 & 5 & 2,97 & & & & \\
\hline & Total & 1087,636 & 10 & & & & & \\
\hline
\end{tabular}

${ }^{1}$ Soma dos Quadrados dos erros; ${ }^{2}$ Graus de liberdade; ${ }^{3}$ Média das somas dos quadrados dos erros. Fonte: Autores (2017) 


\section{REA - Revista de estudos ambientais (Online) v.19, n. 2, p.31-39, jul./dez. 2017}

Como o $\mathrm{F}$ calculado é maior do que o $F$ tabelado, e o p-valor é menor do que 0,05 para ambos os comprimentos de onda, 0 modelo é válido e foi possível gerar uma superfície resposta a fim de que se obtenham os coeficientes do modelo matemático para cada comprimento de onda analisado. A superfície de resposta, gerada com os dados experimentais de tempo, corrente e remoção da cor para o comprimento de $515 \mathrm{~nm}$ pode ser observada na Figura 1.

Os coeficientes obtidos no delineamento estão discriminados na equação 6, referentes ao comprimento de 515 $\mathrm{nm}$, que representa matematicamente a superfície gerada.

$y=57,7+6,6 i-5,2 i^{2}+7,3 t-1,9 t^{2}+4,25 i$

A Figura 2 apresenta a superfície de resposta gerada para o comprimento de 543 $\mathrm{nm}$ a partir dos parâmetros de tempo corrente e remoção de cor.

Figura 1 - Superfície de resposta para o comprimento de 515 nm

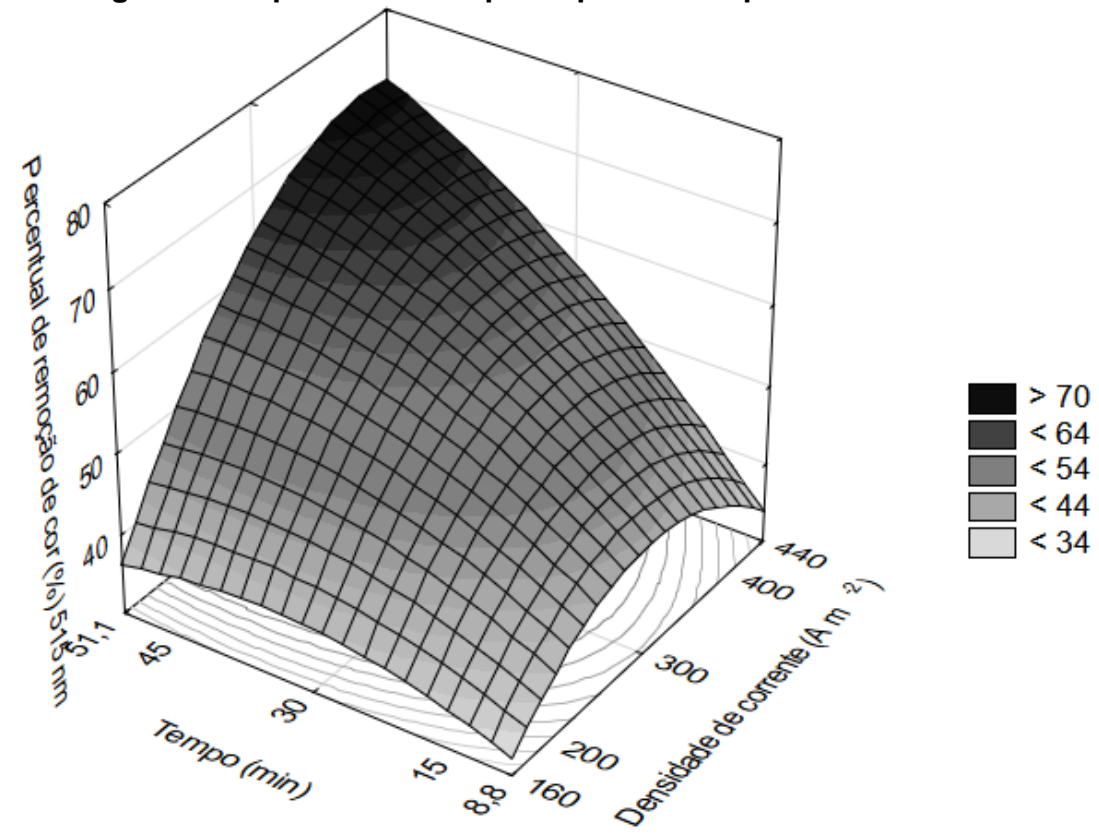

Fonte: Autores (2017)

Os coeficientes do modelo matemático para o comprimento de $543 \mathrm{~nm}$ estão representados na equação 7 .

$y=64,3+6,8 i-5,26 i^{2}+7,9 t-2,41 t^{2}+3,2 i t$

Ao se observar as superfícies de respostas (Figuras 1 e 2) percebe-se que as regiões ótimas de tempo e corrente que resultam na maior remoção de cor, para o comprimento de onda de $515 \mathrm{~nm}$ e $543 \mathrm{~nm}$, são de $360-440 \mathrm{~A} \mathrm{~m}^{-2}$ e de 47-51,1 minutos, respectivamente. São valores próximos, pois as análises de remoção da cor foram realizadas em bandas muito próximas e que possuem intensidade de absorção semelhante. O percentual de remoção de cor ficou na faixa de $70-75 \%$ para comprimento de $515 \mathrm{~nm}$ e de $75-80 \%$ para o comprimento de $543 \mathrm{~nm}$. Souza et al. (2016) trabalhando com eletrocoagulação em efluente têxtil encontraram valores de remoção de cor de até aproximadamente $60 \%$ para um tempo operacional de 50 minutos e intensidade de corrente de $5 \mathrm{~A}$.

Palácio et al. (2015), também avaliando a eletrocoagulação em efluentes têxteis, encontraram valores de remoção de $95 \%$ da cor, utilizando eletrodos de ferro, com um tempo operacional de 60 minutos e uma intensidade de corrente também de $5 \mathrm{~A}$. Diante dessas informações e observando as superfícies de resposta, possivelmente se a faixa de estudo do tempo operacional e intensidade de corrente fossem mais amplas os percentuais de remoção da cor seriam mais altos.

Para a validação do modelo de DCCR foram realizados dois testes em duplicata, usando as condições ótimas de tempo 


\section{REA - Revista de estudos ambientais (Online) v.19, n. 2, p.31-39, jul./dez. 2017}

máximo avaliado de 51,1 minutos $\mathrm{e}$ densidade de corrente de $360 \mathrm{~A} \mathrm{~m}^{-2}$, para cada comprimento de onda estudado, e foram analisadas as respectivas remoções de cor em cada teste e comparadas com a remoção apontada pelo modelo. Também foi analisado qual o consumo de eletrodos e qual o consumo de energia para cada teste. Os resultados dos testes de validação do modelo encontram-se na Tabela 6.

Figura 2 - Superfície de resposta para o comprimento de 543 nm

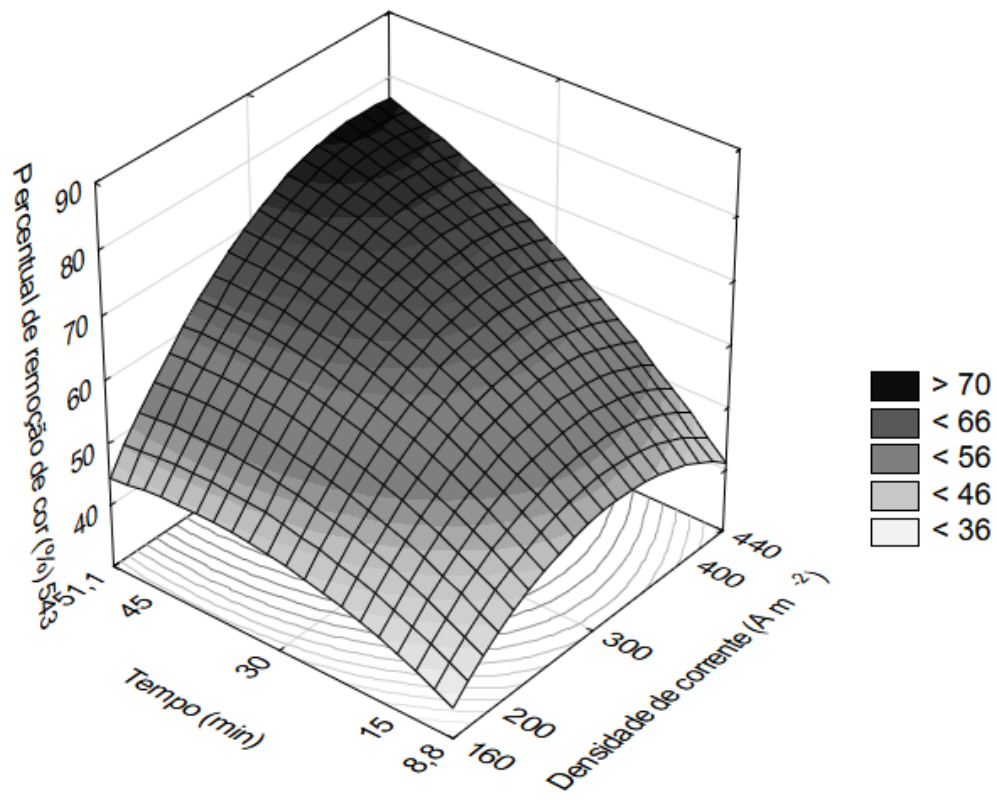

Fonte: Autores (2017)

Tabela 6 - Remoção da cor, consumo de energia e de eletrodos na condição ótima de tratamento

\begin{tabular}{cccccccc}
\hline Testes & $\begin{array}{c}\text { Comprimento } \\
\text { de Onda } \\
(\mathrm{nm})\end{array}$ & $\begin{array}{c}\text { Densidade } \\
\text { de } \\
\text { Corrente } \\
\left(\mathrm{A} \mathrm{m}^{-2}\right)\end{array}$ & $\begin{array}{c}\text { Tempo } \\
(\mathrm{min})\end{array}$ & $\begin{array}{c}\text { Remoção } \\
(\%)\end{array}$ & $\begin{array}{c}\text { Consumo } \\
\text { de energia } \\
\left(\mathrm{Wh} \mathrm{m}^{-3}\right)\end{array}$ & $\begin{array}{c}\text { Consumo de } \\
\text { eletrodos } \\
\text { calculado }\left(\mathrm{g} \mathrm{L}^{-}\right. \\
1)\end{array}$ & $\begin{array}{c}\text { Consumo de } \\
\text { eletrodos } \\
\text { medido }\left(\mathrm{g} \mathrm{L}^{-1}\right)\end{array}$ \\
\hline 1 & 515 & 0,96 & 51,1 & $77,5 \pm 2,5$ & $\begin{array}{c}0,0077 \pm \\
0,0001\end{array}$ & 1,71 & $1,735 \pm 0,005$ \\
2 & 543 & 0,97 & 51,1 & $82 \pm 2$ & $\begin{array}{c}0,0073 \pm \\
0,0005\end{array}$ & 1,73 & $1,75 \pm 0,02$ \\
\hline
\end{tabular}

Fonte: Autores (2017)

A análise dos valores de remoção encontrados nos testes de validação permite inferir que o modelo é válido e existe uma remoção de cor um pouco maior que a teórica calculada. Em relação ao consumo dos eletrodos, os valores calculados e medidos foram muito próximos, ou seja, indica-se a alta confiabilidade da equação usada.

As equações do custo operacional seguiram o modelo da Equação 3 e foram obtidas pela média entre os consumos de energia para 0 teste 1 , correspondente ao comprimento de $515 \mathrm{~nm}$ (Equação 8), e também para os testes 2 (Equação 9) da Tabela 6, que são em relação ao $543 \mathrm{~nm}$.

$$
\begin{aligned}
& C_{0515}=a 0,0077+b 1,71 \\
& C_{0543}=a 0,0073+b 1,73
\end{aligned}
$$

Segundo ABRADEE (2016) a tarifa de energia elétrica é de aproximadamente $R \$$ 0,48 por $\mathrm{kWh}$ e o custo do $\mathrm{kg}$ de ferro é cotado como $3,55 \mathrm{~kg}^{-1}$ (PORTAL METALICA, 2014). Assim para o tratamento de $1 \mathrm{~m}^{3}$ de efluente o custo é estimado em $R \$ 6,07$ para a equação 8 e $R \$ 6,14$ para a equação 9. Pauli et al. (2012) trabalhando com eletrocoagulação de efluente de frigorífico e eletrodos de alumínio chegaram a um valor de aproximadamente $\mathrm{R} \$ 10,00 \mathrm{~m}^{-3}$ efluente para um tempo de tratamento de 50 minutos, 


\title{
REA - Revista de estudos ambientais (Online) \\ v.19, n. 2, p.31-39, jul./dez. 2017
}

quase o dobro do valor encontrado neste estudo.

Cabe ressaltar que $0 \mathrm{pH}$ não foi ajustado para os ensaios, isso porque segundo Pessoa (2008) quando a condutividade é alta o efeito do $\mathrm{pH}$ não é significante para o tratamento e como foi adicionado sal a solução antes do tratamento a condutividade do efluente sintético era alta. Mesmo com o consumo de energia e de eletrodos, a eletrocoagulação apresenta algumas vantagens em relação a outras técnicas como a utilização de pouco espaço e tempo de tratamento relativamente pequeno, se comparado com as lagoas de tratamento. Mesmo com remoções de cor de $70 \%$ existe a possibilidade de reutilização da água tratada dentro da empresa, em banheiros e para a limpeza do ambiente. Além disso, para um sistema mais sustentável e menos oneroso economicamente, pode-se utilizar a energia proveniente de placas fotovoltaicas.

\section{Conclusões}

Os resultados encontrados para a aplicação de eletrofloculação na solução de corante de $50 \mathrm{mg} \mathrm{L}^{-1}$ foram na faixa de 47 51,1 minutos e $360-440 \mathrm{~A} \mathrm{~m}^{-2}$ para os comprimentos de onda estudados.

Os valores de remoção de cor encontrados pelo modelo, que ficaram entre $70-80 \%$, foram validados experimentalmente e foram encontrados valores de $77,5-82 \%$ de remoção, acima dos valores teóricos, o que aponta a eficiência do modelo. Como os valores encontrados de tempo e densidade de corrente para os dois comprimentos de onda analisados foram quase iguais (360-440 A m2 e de 47-51,1 minutos) as mesmas condições (360 $\mathrm{A} \mathrm{m}^{-2}$ e de 51,1 minutos) podem ser utilizadas para o tratamento do efluente sintético.

O consumo de eletrodos e de energia nos tempos e correntes ótimos encontrados ficou em $1,71 \mathrm{~g} \mathrm{~L}^{-1}$ e $0,0077 \mathrm{Wh} \mathrm{m}^{-3}$ para o $515 \mathrm{~nm}$ e $1,73 \mathrm{~g} \mathrm{~L}^{-1}$ e $0,0073 \mathrm{Wh} \mathrm{m}^{-3}$ para o $543 \mathrm{~nm}$.

\section{Application of the electrocoagulation technique for the removal of textile dye}

\begin{abstract}
The objective of this work was to determine the operational time and the electric current density to optimize the color removal from synthetic effluent containing reactive red dye using Rotational Central Compound Design (RCCD). From the optimized conditions, an analysis of operating costs was performed as a function of the spent energy and of the electrode consumption. Optimum conditions and operating costs were determined for wavelengths of 515 and $543 \mathrm{~nm}$. The optimum removal region for the two wavelengths was between $47-51.1$ minutes and 360-440 A $\mathrm{m}^{-}$ 2 , with theoretical removal values estimated at 70-80\%. Electrode and energy consumption at the times and optimized current density found was $1.71 \mathrm{~g} \mathrm{~L}^{-1}$ and $0.0077 \mathrm{Wh} \mathrm{\textrm {m } ^ { - 3 }}$ for the wavelength 515 $\mathrm{nm}$ and $1.73 \mathrm{~g} \mathrm{~L}^{-1}$ and $0.0073 \mathrm{Wh} \mathrm{m}^{-3}$ for $543 \mathrm{~nm}$, resulting in equations: " $C=a 0.0077+b 1.71$ " for the length of $515 \mathrm{~nm}$ and " $C=a 0.0073+b 1,73$ " for the $543 \mathrm{~nm}$, in which a corresponds to the cost of energy (in $R \$ \mathrm{kWh}^{-1}$ ) and b to the mass cost of the plate (in $R \$ \mathrm{~kg}$ electrode $\mathrm{e}^{-1}$ ).
\end{abstract}

Keywords: Current density. Textile dye. Optimization. Operating time.

\section{Referências}

ABRADEE- Associação Brasileira de Distribuidores de Energia Elétrica. Estudo comparativo de Tarifas 2016. 2016. Disponível em: $<\quad$ http://www.abradee.com.br/escolha-abradeepara-voce/material-de-divulgacao/3235-estudocomparativo-de-tarifas-2016-ref-2015-abradee > Acesso em: 06 mar. 2018.

ALEXANDRE, J. E.C.; PARENTE, T. C.; RIBEIRO, J. P.; MAIA, L. G.C.; ABDALA NETO, E. F.; NASCIMENTO, R. F. Avaliação da eficiência da eletrocoagulação-flotação na descoloração de efluente têxtil. $X X$ Congresso Brasileiro de Engenharia Química COBEQ, 2014. Anais ... Florianópolis: 2014

AQUINO NETO, S.; MAGRI, T. C.; SILVA, G. M.; ANDRADE, A. R. Tratamento de resíduos de corante por eletrofloculação: um experimento para cursos de graduação em Química. Química Nova, v. 34, n. 8, p.1468-1471. 2011.

CERQUEIRA, A. A.; MARQUES, M. R. C. Avaliação do processo eletrolítico em corrente 


\section{REA - Revista de estudos ambientais (Online) \\ v.19, n. 2, p.31-39, jul./dez. 2017}

alternada no tratamento de água de produção. Química Nova, v. 34, n. 1, p. 59-63. 2011.

CERQUEIRA, A. A. Aplicação da técnica de eletrofloculação no tratamento de efluentes têxteis. Dissertação (Mestrado em Química), Instituto de Química, Universidade do Estado do Rio de Janeiro- UERJ, Rio de Janeiro. 2006.

ELKADY, M.F.; IBRAHIM, AMAL M.; EL-LATIF, M.M. ABD. Assessment of the adsorption kinetics, equilibrium and thermodynamic for the potential removal of reactive red dye using eggshell biocomposite beads. Desalination, v. 278, p. 412423, 2011.

FLECK, L.; TAVARES, M. H. F.; EYNG, E. Utilização da técnica de eletrofloculação para o tratamento de efluentes têxteis: uma revisão. Revista EIXO, Brasília (DF), v.2, n.2, p. 28-36. Julho/Dezembro de 2013.

GOBBI, L.C.A. Tratamento de água oleosa por eletrofloculação. 120 f. Dissertação (Mestrado em Energia), Universidade Federal do Espírito Santo, São Mateus. 2013.

KELM, C. N. P.; LOPES, N. P. S.; PEREIRA, H. D.; SALES, D. C. S.; CAVALCANTI, L. A. P. Aplicação da eletrofloculação no tratamento de efluentes têxteis de Toritama/PE. Congresso Brasileiro de Gestão Ambiental e Sustentabilidade, v. 2, 2014. Congestas. Anais. Congestas: 2014. p. 371-372.

KOBYA, M.; HIZ, H.; SENTURK, E.; AYDINER, C.; DEMIRBAS, $E$. Treatment of potato chips manufacturing wastewater by electrocoagulation. Desalination, v. 190, p. 201-211. 2006

MOLLAH, M.Y.A.; SCHENNACH, R.; PARGA, J. R.; COCKE, D. L., Electrocoagulation (EC): science and applications. Journal of Hazardous Materials, v. 84, p. 29-41. 2001.

NASCIMENTO, I. L.; GOBBI, L. C. A.; PORTO, P. S. S; ROCHA, S. M. S. Eletrofloculação em efluentes oleosos utilizando eletrodos de alumínio. Blucher Chemical Engineering Proceedings, Vassouras (RJ), v. 1, n. 1. Dezembro de 2014.

PALÁCIO, S. M.; FAGUNDES-KLEN, M. R.; OLIVEIRA, C. C.; JULIANA CARLA GARCIA, J. C.; MANENTI, D. R. Combinação dos processos eletrocoagulação e fotocatálise heterogênea no tratamento de um efluente têxtil clorado. ENGEVISTA, v. 17, n. 3, p. 407-420, 2015.

PARENTE, T. C.; SAWAKI, R. V.; ALEXANDRE, J. E. C.; LIMA, A. C.; RIBEIRO, J. P.; ABDALA NETO, E. F.; NASCIMENTO, R. F. validação do modelo de eletrocoagulação flotação no tratamento de efluente têxtil visando à remoção de $\mathrm{DQO}$, utilizando reator em batelada. XI Congresso
Brasileiro de Engenharia Química em Iniciação Científica, 2015. Unicamp, Campinas (SP). Anais. Campinas :19 a 22 de julho de 2015.

PASCHOAL, F. M. M; TREMILIOSI-FILHO, G. Aplicação da tecnologia de eletrofloculação na recuperação do corante índigo blue a partir de efluentes industriais. Química Nova, v. 28, n. 5, p. 766-772, 2005

PAULI, A. R.; ESPINOZA-QUINONES, F. R.; MÓDENES, A. N.; BORBA, C. E.; THEODORO, P. S. Aplicação do planejamento experimental no método de eletro-coagulação para a remoção de matéria orgânica de efluentes frigoríficos. ENGEVISTA, v. 14, n. 3. p. 293-301, dezembro 2012.

PESSOA, G. P. Estudo da remoção de cor de efluente têxtil por eletrocoagulação. Dissertação (Mestrado em Saneamento Ambiental), Universidade Federal do Ceará, Fortaleza. 2008.

PORTAL METÁLICA. Indicadores de preço- perfis. $2014 . \quad$ Disponível em: <http://wwwo.metalica.com.br/cotacao-de-precoperfis>. Acesso em: 23 nov. 2017.

SANTOS, P. K.; KARLA CARVALHO FERNANDES, K. C.; FARIA, L. A.; FREITAS, A. C.; SILVA, L. M. Descoloração e degradação do azo corante vermelho GRLX-220 por ozonização. Química Nova, v. 34, n. 8, p. 1315-1322, 2011.

SENTHILKUMAAR, S.; KALAAMANI, P; PORKODI, K.; VARADARAJAN, P.R.; SUBBURAAM, C.V. Adsorption of dissolved Reactive red dye from aqueous phase onto activated carbon prepared from agricultural waste. Bioresource Technology, v. 97, p. 1618-1625, 2006.

SOUZA, P. C.; PEREIRA, N. C.; GONÇALVES, M. S.; CONSOLIN FILHO, N.; RODRIGUES, P. H.; JAMARIM, V. M. Estudo do tratamento de efluente têxtil através de processos de coagulação/floculação e eletrocoagulação. exacta, Belo Horizonte, v. 9, n. 2, p. 123-132, 2016.

VALERO, D; ORTIZ, J. M; EXPÓSITO, E; MONTIE, V; ALDAZ, A. Electrocoagulation of a synthetic textile effluent powered by photovoltaic energy without batteries: Direct connection behavior. Solar Energy Materials \& Solar Cells, v. 92, p. 291-297. 2008.

VASQUES, A. R; SOUZA, S. M. A. G. U., WEISSENBERG, L.; SOUZA, A. A. U.; VALLE, J. A. B. Adsorção dos corantes RO16, RR2 e RR141 utilizando lodo residual da indústria têxtil. Engenharia Sanitária e Ambiental, Rio de Janeiro, v.16, n.3, p. 245-252, jul./set. 2011. 